OPEN ACCESS

Edited by:

Martin Burtscher,

University of Innsbruck, Austria

Reviewed by:

Stephen Seiler,

University of Agder, Norway

Caio Victor Sousa

Northeastern University, United States

*Correspondence:

Bergita Ganse

bergita.ganse@uks.eu

Specialty section:

This article was submitted to

Exercise Physiology,

a section of the journal

Frontiers in Physiology

Received: 25 June 2021

Accepted: 30 August 2021

Published: 28 September 2021

Citation:

Ganse B, Braczynski AK, Hoog Antink C, Knobe M, Pohlemann T and

Degens $H$ (2021) Acceleration of

Longitudinal Track and Field Performance Declines in Athletes Who Still Compete at the Age of 100 Years.

Front. Physiol. 12:730995

doi: 10.3389/fphys.2021.730995

\section{Acceleration of Longitudinal Track and Field Performance Declines in Athletes Who Still Compete at the Age of 100 Years}

\author{
Bergita Ganse ${ }^{1,2 *}$, Anne Kristin Braczynski ${ }^{3,4}$, Christoph Hoog Antink ${ }^{5}$, Matthias Knobe ${ }^{6}$, \\ Tim Pohlemann ${ }^{2}$ and Hans Degens ${ }^{7,8}$
}

${ }^{1}$ Division of Surgery, Werner Siemens Foundation Endowed Chair of Innovative Implant Development, Saarland University, Homburg, Germany, ${ }^{2}$ Department of Trauma, Hand and Reconstructive Surgery, Saarland University, Homburg, Germany, ${ }^{3}$ Department of Neurology, RWTH Aachen University Hospital, Aachen, Germany, ${ }^{4}$ Institut für Physikalische Biologie, Heinrich-Heine University Düsseldorf, Düsseldorf, Germany, ${ }^{5} K I S^{*} M E D$ - Al Systems in Medicine, Electrical Engineering and Information Technology, TU Darmstadt, Darmstadt, Germany, ${ }^{6}$ Department of Orthopaedic and Trauma Surgery, Lucerne Cantonal Hospital, Lucerne, Switzerland, ${ }^{7}$ Research Centre for Musculoskeletal Science \& Sports Medicine, Manchester Metropolitan University, Manchester, United Kingdom, ${ }^{8}$ Institute of Sport Science and Innovations, Lithuanian Sports University, Kaunas, Lithuania

While physical performance decline rates accelerate after around the age of 70 years, longitudinal athletic performance trends in athletes older than 95 years are unknown. We hypothesized a further accelerated decline in human performance in athletes who still perform at the age of 100 years. To investigate this, longitudinal data of all athletes with results at or over the age of 100 years were collected from the "World Master Rankings" data base spanning 2006-2019 (138 results from 42 athletes; 5 women, 37 men; maximum 105 years) and compared to previously published longitudinal data from 80- to 96-year-old athletes from Sweden (1,134 results from 374 athletes). Regression statistics were used to compare performance decline rates between disciplines and age groups. On average, the individual decline rate of the centenarian group was 2.53 times as steep (100 m: 8.22x; long jump: 0.82x; shot put: 1.61x; discus throw: 1.04x; javelin throw: $0.98 \mathrm{x}$ ) as that seen in non-centenarians. The steepest increase in decline was found in the 100-m sprint ( $t$-test: $p<0.05$, no sign. difference in the other disciplines). The pooled regression statistics of the centenarians are: $100 \mathrm{~m}: R=0.57, p=0.004$; long jump: $R$ $=0.90, p<0.001$; shot put: $R=0.65, p<0.001$; discus throw: $R=0.73, p<0.001$; javelin throw: $R=0.68, p<0.001$. This first longitudinal dataset of performance decline rates of athletes who still compete at 100 years and older in five athletics disciplines shows that there is no performance plateau after the age of 90 , but rather a further acceleration of the performance decline.

Keywords: aging, master athletics, physical activity, longevity, oldest-old, centenarian, javelin throw, long jump 


\section{INTRODUCTION}

Human longevity, limits of the human life span and physical performance in old age are of great interest due to the increasing proportion of older people in western societies. It has been speculated that the human mortality rate reaches a "plateau" after the age of 105 (Barbi et al., 2018) but it is unknown whether a plateau, or attenuated decline, also exists for physical performance. Frailty that is associated with mobility limitations and loss of autonomy is a major cause of a poor quality of life of the oldest old (Portegijs et al., 2016; Ding et al., 2017). Indeed, for the oldest old, key to a good quality of life is physical independence (Arai et al., 2014). Data of the few extraordinary individuals who are still able to compete in sports at an age where most people are unable to move and care for themselves are therefore of particular interest for the older people themselves, healthcare providers and insurers, as they may not only inspire other older people, but also show the limits of the physiologically achievable in the oldest old.

In the master athlete population, performance decline rates based on results from annual rankings are currently known up until the age of 95, but not beyond (Ganse et al., 2020a). Analyses of such databases have revealed that the rate of decline of physical performance is initially almost linear, and then accelerates from around the age of 70 years ( $\mathrm{Da}$ Silva Aguiar et al., 2020). This acceleration has not only been shown in crosssectional (Young and Starkes, 2005; Ganse et al., 2018), but also in longitudinal data (Lazarus and Harridge, 2017; Ganse et al., 2020b). The decline rate accelerates even further beyond the age of 80 (Ganse et al., 2020b). However, while in longitudinal data most individuals show an acceleration, some athletes have a slower or faster performance decline than others (Donato et al., 2003; Rubin et al., 2013; Ganse et al., 2020b; Hoog Antink et al., 2021). Only few cross-sectional data of centenarian athletes are available (Lepers et al., 2016), but there are no longitudinal data on athletes around 100 years, despite the need for more knowledge in the field of frailty and aging-research (Arai et al., 2014; Portegijs et al., 2016; Ding et al., 2017). Increased participation of centenarians in competitive sports now allows us to assess the rate of performance decline also in a population of oldest-old athletes.

Based on the observation that world records up to the age of 105 years suggest a progressive age-related acceleration of performance decline in many disciplines (Baker and Tang, 2010), we hypothesized a further acceleration of the decline rate in performance of master athletes that are around 100 years of age. To determine whether the rate of performance decline in master athletes is accelerated after the age of 90 years, we combined the data from $80+$-year-old athletes in 5 disciplines from a longitudinal data set we published recently (Ganse et al., 2020b) with new longitudinal data of athletes who still competed at age 100 years or older from the database "World Master Rankings." This represents the first master athletics performance data set ever published of this age group.

\section{MATERIALS AND METHODS}

Ethical approval was obtained from the IRB of Saarland Medical Board (Ärztekammer des Saarlandes, application number 135/21).

\section{Generation of Data Set}

Performance data of all athletes with results at age 100 years and older were collected from the publicly available "World Master Rankings" data base (www.mastersrankings.com). As the data base reached back to the year 2006, and since restrictions due to the COVID-19 pandemic led to the absence of data in the year 2020, results of both sexes from 2006 to 2019 were considered. For all athletes who had a result at an age of 100 years and older, the data base was searched for additional results from previous ages going back to as early as the age of 90 years to obtain a longitudinal trajectory of the individual.

\section{The Swedish Data Set}

The new data were compared to the data of all $80+$-year-old athletes in a data set from Sweden that was published previously (Ganse et al., 2020b). Briefly, this data set comprises all data from the Swedish master athletics rankings from the years 1901 to 2019 and is the largest longitudinal master athletics data set published to date.

\section{Implements}

Changes in weights of javelins, discusses, and shots with age did not affect the results in the present analysis, as implement weights in the throwing disciplines stay constant for athletes 80 years and older. The following implements are used by athletes 80 years and older: shot put: men $3 \mathrm{~kg}$, women $2 \mathrm{~kg}$; discus throw: men $1 \mathrm{~kg}$, women $750 \mathrm{~g}$; javelin throw: men and women $400 \mathrm{~g}$.

\section{Statistical Analysis}

All statistical tests were executed with IBM SPSS Statistics version 25. Regression statistics were employed to compare performance decline rates between disciplines and age groups. Regression coefficients and their corresponding $p$-values were calculated (significance level 0.05). Disciplines were included in the study if the following inclusion criteria were met: at least three athletes with at least two results in the data set and at least one result at an age of 100 years or more. Linear regression decline rates were computed for the younger (the data from Sweden) and the older (the data of the athletes who still compete at 100 years) data set separately to allow direct comparison of declines, even though other regression types would have delivered higher $R^{2}$-values. Two separate analyses were conducted: linear regression on all pooled data points of each group, and linear regression on the individual regression lines of each athlete, combined with $t$-tests. We chose linear regression, as the optimal type of regression function differed between disciplines, and to be able to compare decline slopes between the centenarians and the non-centenarian old athletes. 
TABLE 1 | Numbers of athletes and data points per discipline in the present centenarian (100+-years group) and Swedish (80+ group) data sets, separated by sex.

\begin{tabular}{|c|c|c|c|c|c|c|}
\hline \multirow[b]{2}{*}{ Discipline } & \multicolumn{3}{|c|}{ Number of centenarian athletes } & \multicolumn{3}{|c|}{ Number of centenarian data points } \\
\hline & Men & Women & Total & Men & Women & Total \\
\hline $100 \mathrm{~m}$ & 7 & 3 & 10 & 18 & 6 & 24 \\
\hline Long jump & 3 & 0 & 3 & 10 & 0 & 10 \\
\hline Shot put & 12 & 1 & 13 & 36 & 2 & 38 \\
\hline Discus throw & 9 & 0 & 9 & 39 & 0 & 39 \\
\hline Javelin throw & 6 & 1 & 7 & 23 & 4 & 27 \\
\hline \multirow[t]{2}{*}{ Sum } & 37 & 5 & 42 & 126 & 12 & 138 \\
\hline & \multicolumn{3}{|c|}{ Number of athletes 80+ years (Sweden) } & \multicolumn{3}{|c|}{ Number of data points $80+$ years (Sweden) } \\
\hline Discipline & Men & Women & Total & Men & Women & Total \\
\hline $100 m$ & 51 & 4 & 55 & 94 & 11 & 105 \\
\hline Long jump & 31 & 5 & 36 & 83 & 10 & 93 \\
\hline Shot put & 81 & 16 & 97 & 290 & 35 & 325 \\
\hline Discus throw & 91 & 18 & 109 & 307 & 41 & 348 \\
\hline Javelin throw & 68 & 9 & 77 & 248 & 15 & 263 \\
\hline Sum & 322 & 52 & 374 & 1,022 & 112 & 1,134 \\
\hline
\end{tabular}

\section{RESULTS}

\section{Characterization of the Data Sets}

We collected 138 results from 42 athletes (5 women and 37 men) of whom results at the age of 100 years or more up to the age of 105 were present in the data base (Table 1). The most data in this age group were available from discus throw and shot put, followed by javelin throw, 100-m sprint and long jump. Table 1 also shows the numbers of athletes and results in the disciplines in the other longitudinal data set of athletes between 80 and 96 years of age with 1,134 results from 374 athletes.

\section{Decline Rates}

Figures 1A-E and Table 2 show that in all five disciplines the average decline rate was higher in the older compared to the younger athletes. The steepest increase in performance decline was found in the 100 -m sprint ( $t$-test, $p<0.05)$, where the decline rate was $8 \mathrm{x}$ as steep in the older compared to the younger group (Table 2). The least steep increase in performance decline was found in the javelin throw. The increases in decline were not significant in long jump and in the throws ( $t$-test, Table 2). On average, the decline rate of centenarians was $2.53 \mathrm{x}$ as steep as compared to that between 80 and 96 years. Figure 1F shows a direct comparison of the disciplines.

\section{Sex Differences}

Only $13.5 \%$ (5 out of 42 athletes) among the centenarians were women, which is in line with the $13.9 \%$ (52 out of 374 athletes) in the Swedish data set. Three women had results in the $100-\mathrm{m}$ sprint, one in the shot put and one in the javelin throw. Due to the low number of women in the data-set, an analysis of sex differences in decline rates was not possible.

\section{DISCUSSION}

In the present study, we analyzed performance declines in five athletic disciplines of 42 master athletes who still performed at 100 years of age and older, and compared the data to the $80+-$ year-old athletes from a longitudinal data set we have published previously (Ganse et al., 2020b). The main finding of the study was that performance decline rates of centenarian athletes were on average 2.53-times as steep as the decline rate between 80 and 96 years of age. The fastest drop occurred in the $100-\mathrm{m}$ sprint, while the javelin throw showed the least steep increase in the slope of decline. Of the centenarians, $13.5 \%$ were women, which is in line with the $13.9 \%$ in the younger collective.

\section{Performance Trends in Aging}

It has been reported that the rate of performance decline was more than three times as fast in athletes older than 80 years compared to 30- to 69-year-old athletes (Ganse et al., 2020a,b). Accelerated decline rates after the age of 70 have also been shown in other disciplines and sports (Young and Starkes, 2005; Berthelot et al., 2012; Rubin et al., 2013; Dahl et al., 2020). The "plateau" in mortality rate in the oldest old (Barbi et al., 2018) may suggest a stabilization of the age-related rate of decrease in physical decline. Our data, however, do not show evidence for such a plateau in physical performance, but rather that the rate of decline is accelerated even further in 90+-year-old athletes beyond that seen in 80+-year-old athletes. Ongoing increases in decline rates were also previously reported from master world records (Baker and Tang, 2010; Akkari et al., 2015). At the same time, our data suggest that there is variation in the decline rate between individuals, that was also shown in the Swedish data set (Hoog Antink et al., 2021). Understanding the underlying factors for these differences could help to develop and improve interventions to slow down performance decline rates.

Differences in decline rates between track and field disciplines may be explained by specific skills required in each discipline, 


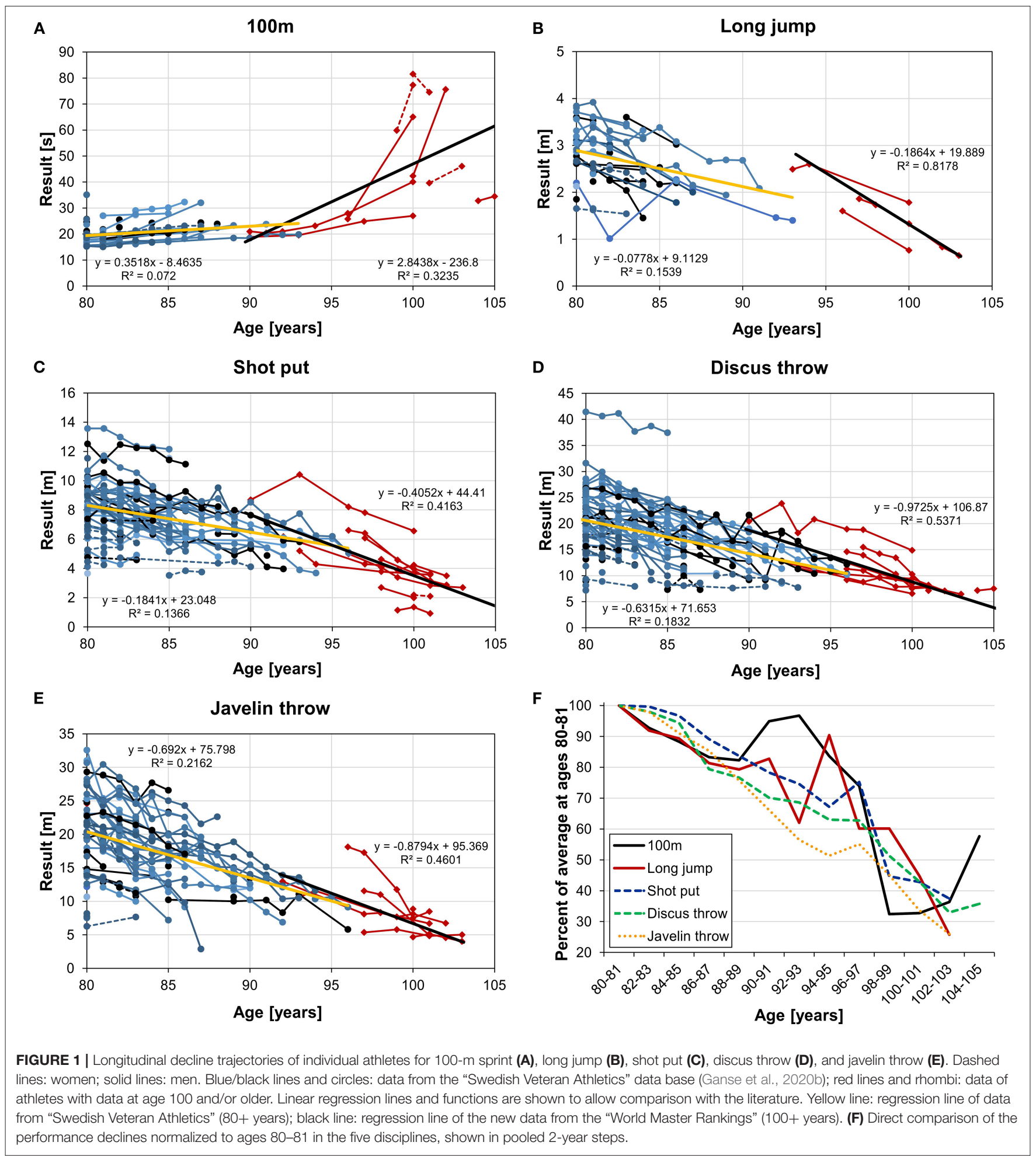

such as power, speed, agility, coordination or endurance, that may show different rates of age-related decline. For instance, the fastest performance decline was observed in the $100-\mathrm{m}$ sprint that requires mainly speed and power (Dahl et al., 2019), while the javelin throw showed the least steep decline rate and depends besides power, on agility and coordination (Ganse and Degens, 2018). This needs to be studied in more detail, however, as previously we have observed that there was no significant difference in the rate of decline in aerobic and anaerobic power in master athletes (Bagley et al., 2019). It is 
TABLE 2 | Comparison of decline rates between the 80+-years-old and centenarian group in seconds or meters per year and the ratio.

\begin{tabular}{|c|c|c|c|c|c|c|c|c|c|c|c|}
\hline \multirow[b]{2}{*}{ Discipline } & \multirow[b]{2}{*}{ R 80+ } & \multicolumn{3}{|c|}{$\begin{array}{l}\text { Linear regression, all data } \\
\text { points pooled }(\mathbf{R})\end{array}$} & \multicolumn{3}{|c|}{$\begin{array}{l}\text { Decline rates all data } \\
\text { points pooled (slopes) }\end{array}$} & \multicolumn{4}{|c|}{$\begin{array}{l}\text { Average individual } \\
\text { decline rates (slopes) }\end{array}$} \\
\hline & & $\begin{array}{c}\text { R } \\
\text { Centenarians }\end{array}$ & p 80+ & $\begin{array}{c}p \\
\text { Centenarians }\end{array}$ & $80+$ group & Centenarians & Ratio & $80+$ group & Centenarians & Ratio & $\begin{array}{l}\text { P-value } \\
\text { (t-test) }\end{array}$ \\
\hline $100 \mathrm{~m}$ & 0.27 & 0.57 & 0.006 & 0.004 & 0.35 & 2.84 & 8.08 & $0.68 \pm 0.86$ & $5.59 \pm 8.42$ & 8.22 & $<0.05$ \\
\hline Long jump & 0.39 & 0.90 & $<0.001$ & $<0.001$ & 0.08 & 0.19 & 2.40 & $0.22 \pm 0.45$ & $0.18 \pm 0.06$ & 0.82 & 0.43 \\
\hline Shot put & 0.38 & 0.65 & $<0.001$ & $<0.001$ & 0.18 & 0.51 & 2.74 & $0.23 \pm 0.26$ & $0.37 \pm 0.21$ & 1.61 & 0.06 \\
\hline Discus throw & 0.43 & 0.73 & $<0.001$ & $<0.001$ & 0.63 & 0.97 & 1.54 & $0.79 \pm 1.14$ & $0.82 \pm 0.70$ & 1.04 & 0.47 \\
\hline Javelin throw & 0.47 & 0.68 & $<0.001$ & $<0.001$ & 0.69 & 0.88 & 1.27 & $0.94 \pm 0.67$ & $0.92 \pm 0.88$ & 0.98 & 0.48 \\
\hline Average & & & & & & & 3.21 & & & 2.53 & \\
\hline
\end{tabular}

The $R$ and slope values were calculated on all data points pooled. "Average individual decline rates" were derived as the average of the individual regression slopes.

clear that different physiological systems contribute to the success in different disciplines, where for instance power events are particularly dependent on muscle power, and endurance events are most limited by the cardiovascular system. It is therefore of interest to assess to what extent performance declines in different disciplines are attributable to proportional decrements in the muscle, respiratory (Degens et al., 2013), or cardiovascular function (Ganse and Degens, 2021).

\section{Centenarian Disciplines}

Data from the oldest-old athletes were only available in 1 of the 10 individual running events, 1 of the 4 jumping events, but 3 of the 5 throwing events contested at the International Masters Games ${ }^{1}$. Interestingly, the event selection of athletes changes in the oldest old compared to earlier ages. Perhaps these disciplines were chosen, as the oldest-old athletes are physically unable to compete in other events. Such a limitation may already occur before the age of 100 years, as in another data set none of the 80-94-year-old athletes participated in hurdles or in other jumps (Ganse et al., 2020a). Possible factors that may contribute to the absence of oldest-old competitors in theses disciplines may be lack of sufficient muscle power and/or fear of injury.

\section{Sex Differences}

Despite the higher life expectancy of women ${ }^{2}$, only $13.5 \%$ of the centenarian athletes in the present study were women. In comparison, $22 \%$ of the athletes in the largest longitudinal master athletics data set were women (Ganse et al., 2020b). Among the athletes with results at age 80 and older from the same data set, however, only $13.9 \%$ were women. The low proportion of oldestold women may be related to a lower participation of women in sports in the past, where changes in society over the decades have stimulated a growing interest in sports and fitness in women, explaining a larger proportion of competing women at younger ages (O’Brien and Robertson, 2010).

\section{Strengths and Weaknesses}

The main strength of the present study is that it is the first to present longitudinal data on changes in performance after

\footnotetext{
$\overline{{ }^{1} \text { https://imga.ch/masters-sports/sports-and-disciplines/athletics/ }}$

2 "Human Development Report”, United Nations Development Program. Available at http://hdr.undp.org/en/content/2019-human-development-index-ranking.
}

the age of 95 of athletes still competing at the age of 100 or over. The main weakness is that a low amount of data from women led to the inability to test for sex differences. This is particularly interesting, as differences in decline rates between men and women have been shown in previous studies (Ganse et al., 2018; Gava et al., 2020). Larger data sets may show that the increases in decline between the younger and the older group are actually significant.

\section{Conclusions}

We presented the first data set of performance decline rates after the age of 90 of athletes still competing at the age of 100 or over in five track and field disciplines. In this age group, the agerelated performance decline was even faster than that previously reported in 80+-year-old athletes.

\section{DATA AVAILABILITY STATEMENT}

Publicly available datasets were analyzed in this study. This data can be found at: www.mastersrankings.com.

\section{ETHICS STATEMENT}

Written informed consent was not obtained from the individual(s) for the publication of any potentially identifiable images or data included in this article.

\section{AUTHOR CONTRIBUTIONS}

BG contributed the idea and worked on data analysis and interpretation, figures, tables, drafting, and manuscript submission. HD contributed to the statistical analysis and data discussion. $\mathrm{AB}, \mathrm{CHA}, \mathrm{MK}$, and $\mathrm{TP}$ helped with data interpretation. All authors have contributed to manuscript drafting and revision, read, and approved the submitted version of the manuscript.

\section{FUNDING}

BG was funded by the German Research Foundation (DFG, grant number GA 2420/1-1). 


\section{REFERENCES}

Akkari, A., Machin, D., and Tanaka, H. (2015). Greater progression of athletic performance in older Masters athletes. Age Ageing 44, 683-686. doi: 10.1093/ageing/afv023

Arai, Y., Inagaki, H., Takayama, M., Abe, Y., Saito, Y., Takebayashi, T., et al. (2014). Physical independence and mortality at the extreme limit of life span: supercentenarians study in Japan. J. Gerontol. A Biol. Sci. Med. Sci. 69, 486-494. doi: 10.1093/gerona/glt146

Bagley, L., McPhee, J. S., Ganse, B., Muller, K., Korhonen, M. T., Rittweger, J., et al. (2019). Similar relative decline in aerobic and anaerobic power with age in endurance and power master athletes of both sexes. Scand. J. Med. Sci. Sports 29, 791-799. doi: 10.1111/sms.13404

Baker, A. B., and Tang, Y. Q. (2010). Aging performance for masters records in athletics, swimming, rowing, cycling, triathlon, and weightlifting. Exp. Aging Res. 36, 453-477. doi: 10.1080/0361073X.2010.507433

Barbi, E., Lagona, F., Marsili, M., Vaupel, J. W., and Wachter, K. W. (2018). The plateau of human mortality: demography of longevity pioneers. Science 360, 1459-1461. doi: 10.1126/science.aat3119

Berthelot, G., Len, S., Hellard, P., Tafflet, M., Guillaume, M., Vollmer, J. C., et al. (2012). Exponential growth combined with exponential decline explains lifetime performance evolution in individual and human species. Age 34, 1001-1009. doi: 10.1007/s11357-011-9274-9

Da Silva Aguiar, S., Sousa, C. V., Sales, M., M., Sousa, H. G., Santos, P. A., et al. (2020). Age-related decrease in performance of male masters athletes in sprint, sprint-endurance, and endurance events. Sport Sci. Health. 16, 385-392. doi: 10.1007/s11332-019-00613-6

Dahl, J., Degens, H., Hildebrand, F., and Ganse, B. (2019). Age-related changes of sprint kinematics. Front. Physiol. 10:613. doi: 10.3389/fphys.2019.00613

Dahl, J., Degens, H., Hildebrand, F., and Ganse, B. (2020). Do changes in middle-distance running kinematics contribute to the age-related decline in performance? J. Musculoskelet. Neuronal Interact. 20, 94-100.

Degens, H., Rittweger, J., Parviainen, T., Timonen, K. L., Suominen, H., Heinonen, A., et al. (2013). Diffusion capacity of the lung in young and old endurance athletes. Int. J. Sports Med. 34, 1051-1057. doi: 10.1055/s-0033-1345137

Ding, Y. Y., Kuha, J., and Murphy, M. (2017). Pathways from physical frailty to activity limitation in older people: identifying moderators and mediators in the English Longitudinal Study of Ageing. Exp. Gerontol. 98, 169-176. doi: 10.1016/j.exger.2017.08.029

Donato, A. J., Tench, K., Glueck, D. H., Seals, D. R., Eskurza, I., and Tanaka, H. (2003). Declines in physiological functional capacity with age: a longitudinal study in peak swimming performance. J. Appl. Physiol. 94, 764-769. doi: 10.1152/japplphysiol.00438.2002

Ganse, B., and Degens, H. (2018). Accelerated decline in javelin throwing performance in master athletes 70 years and older. Sports Med. Int. Open 02, E79-E83. doi: 10.1055/a-0635-0584

Ganse, B., and Degens, H. (2021). Current insights in the age-related decline in sports performance of the older athlete. Int. J. Sports Med. doi: 10.1055/a-1480-7730. [Epub ahead of print].

Ganse, B., Drey, M., Hildebrand, F., Knobe, M., and Degens, H. (2020a). Performance declines are accelerated in the oldest-old track and field athletes 80 to 94 years of age. Rejuvenation Res. 24, 20-27. doi: 10.1089/rej.2020.2337
Ganse, B., Ganse, U., Dahl, J., and Degens, H. (2018). Linear decrease in athletic performance during the human life span. Front. Physiol. 9:1100. doi: 10.3389/fphys.2018.01100

Ganse, B., Kleerekoper, A., Knobe, M., Hildebrand, F., and Degens, H. (2020b). Longitudinal trends in master track and field performance throughout the aging process: 83,209 results from Sweden in 16 athletics disciplines. GeroScience 42, 1609-1620. doi: 10.1007/s11357-020-00275-0

Gava, P., Giuriati, W., and Ravara, B. (2020). Gender differences of aging performance decay rate in normalized Master World Records of Athletics: much less than expected. Eur. J. Transl. Myol. 30:8869. doi: 10.4081/ejtm.2019.8869

Hoog Antink, C., Braczynski, A. K., and Ganse, B. (2021). Learning from Machine Learning: prediction of age-related athletic performance decline trajectories. GeroScience. doi: 10.1007/s11357-021-00411-4. [Epub ahead of print].

Lazarus, N. R., and Harridge, S. D. R. (2017). Declining performance of master athletes: silhouettes of the trajectory of healthy human ageing? J. Physiol. 595, 2941-2948. doi: 10.1113/JP272443

Lepers, R., Stapley, P. J., and Cattagni, T. (2016). Centenarian athletes: examples of ultimate human performance? Age Ageing. 45, 732-736. doi: 10.1093/ageing/afw111

O'Brien, M., and Robertson, A. (2010). Women and sport. Scott. Med. J. 55, 25-28. doi: 10.1258/rsmsmj.55.2.25

Portegijs, E., Rantakokko, M., Viljanen, A., Sipilä, S., and Rantanen, T. (2016). Is frailty associated with life-space mobility and perceived autonomy in participation outdoors? A longitudinal study. Age Ageing 45, 550-553. doi: 10.1093/ageing/afw072

Rubin, R. T., Lin, S., Curtis, A., Auerbach, D., and Win, C. (2013). Declines in swimming performance with age: a longitudinal study of Masters swimming champions. Open Access J Sports Med. 4, 63-70. doi: 10.2147/OAJSM.S 37718

Young, B. W., and Starkes, J. L. (2005). Career-span analyses of track performance: longitudinal data present a more optimistic view of age-related performance decline. Exp. Aging Res. 31, 69-90. doi: 10.1080/03610730590882855

Conflict of Interest: The authors declare that the research was conducted in the absence of any commercial or financial relationships that could be construed as a potential conflict of interest.

Publisher's Note: All claims expressed in this article are solely those of the authors and do not necessarily represent those of their affiliated organizations, or those of the publisher, the editors and the reviewers. Any product that may be evaluated in this article, or claim that may be made by its manufacturer, is not guaranteed or endorsed by the publisher.

Copyright (C) 2021 Ganse, Braczynski, Hoog Antink, Knobe, Pohlemann and Degens. This is an open-access article distributed under the terms of the Creative Commons Attribution License (CC BY). The use, distribution or reproduction in other forums is permitted, provided the original author(s) and the copyright owner(s) are credited and that the original publication in this journal is cited, in accordance with accepted academic practice. No use, distribution or reproduction is permitted which does not comply with these terms. 